\title{
1-YIA-08 YIA
}

\section{2,5-Dimethylcelecoxib attenuates cardiac fibrosis after cryoinjury-induced myocardial infarction by suppressing the fibroblast-myofibroblast differentiation}

\section{Eigo Ikushima $^{1}$, Shin Ishikane ${ }^{1}$, Tomohiro Takiguchi ${ }^{2}$, Yumiko Toyohira $^{1}$, Fumi Takahashi ${ }^{1}$}

${ }^{I}$ Department of Pharmacology, University of Occupational and Environmental Health, Japan, ${ }^{2}$ Dep. Clin. Pharm., Grad. Sch. Med., Kyushu Univ.

\section{【Background】}

Cardiac fibrosis is associated with heart diseases, such as myocardial infarction (MI), and activated fibroblasts (myofibroblasts) play a main role during fibrosis progression. Although we reported that 2,5 dimethylcelecoxib (DM-

C) prevents cardiac fibrosis, the molecular mechanism, including the effect on myofibroblast differentiation, is not clarified yet.

【Objective】

We investigate the effect of DM-C on MI-caused fibrosis and fibroblast-myofibroblast differentiation using in vivo and in vitro models.

\section{【Methods】}

In vivo: Cryoinjury-induced MI (CMI) mouse model was employed. In DM-C group, the mice received DM-C for 4 weeks from 3 days before the operation. Cardiac function was evaluated with transthoracic echocardiography every week. Four weeks after operation, the heart was removed and the fibrosis area was evaluated.

In vitro: The effect DM-C on myofibroblast-differentiation induced by TGF-b using SD rat dermal fibroblast was examined.

【Results】

In DM-C group, the ejection fraction was increased than control group and, according with this, the fibrosis area is reduced. Further, DM-C significantly suppressed aSMA expression (myofibroblast marker) and the phosphorylation level of Akt, GSK-3b and Smad2/3.

\section{【Conclusion】}

These results suggest that DM-C attenuates cardiac fibrosis after MI through inhibition of fibroblast-myofibroblast differentiation. DM-C also inhibited the TGF-b /SMAD2/3 signaling pathway by Akt inhibition. Therefore, DM-C has a potential as the novel drug for treatment of cardiac fibrosis after MI. 